\title{
Histological grading of the prostate carcinoma using deep learning: an unsupervised approach (Erratum)
}

Markus Bauer, Sebastian Zürner, Markus Kreuz, Dominik Otto, Georg Popp, et al.

Markus Bauer, Sebastian Zürner, Markus Kreuz, Dominik Otto, Georg Popp, Ulf-Dietrich Braumann, "Histological grading of the prostate carcinoma using deep learning: an unsupervised approach (Erratum)," Proc. SPIE 11603, Medical Imaging 2021: Digital Pathology, 1160319 (25 June 2021); doi: $10.1117 / 12.2604021$

SPIE. Event: SPIE Medical Imaging, 2021, Online Only 


\section{Histological grading of the prostrate carcinoma using deep learning: an unsupervised approach (Erratum)}

Markus Bauer, Institute of Applied Informatics (InfAI), Sebastian Zürner, Fraunhofer Institute for Cell Therapy and Immunology (IZI), Markus Kreuz, Fraunhofer Institute for Cell Therapy and Immunology (IZI), Georg Popp, Fraunhofer Institute for Cell Therapy and Immunology (IZI), Ulf-Dietrich Braumann, Institute for Applied Informatics (InfAl)

Proceedings Volume 11603, Medical Imaging 2021: Digital Pathology; 116030Y (2021)

https://doi.org/10.1117/12.2581043

Event: Medical Imaging 2021, Digital Forum Online only

Online Publication Date: 15 February 2021

Erratum Published: 24 June 2021

A revised version of this manuscript was published on 24 June 2021 . Details of the revision are provided in the text that accompanies this Erratum. The original paper has been updated. 